\title{
Multi-point Connectivity for Reliable mmWave
}

\author{
Dileep Kumar, Jarkko Kaleva, Antti Tölli \\ Centre for Wireless Communications, University of Oulu, Oulu, Finland \\ Email: \{dileep.kumar, jarkko.kaleva, antti.tolli\}@oulu.fi
}

\begin{abstract}
The fundamental challenge for mmWave communication is the sensitivity of $\mathbf{m m W a v e}$ signals to the blockage, which gives rise to unstable connectivity and impacts the reliability of a system. In this paper, we explore the viability of using coordinated multi-point connectivity, which facilitates multi-user precoding across spatially distributed transmitters, to ensure reliable connectivity even if one or more dominant links are under blockage. We provide successive convex approximation based algorithms for weighted sum-rate maximization and minimum user-rate maximization problems while considering the effects of random link blockage. For the downlink precoder design, a conservative estimate of the available rate per user is computed over all subset combinations of potentially blocked links. The trade-off between achievable sum-rate and reliable connectivity is illustrated via numerical examples. In the presence of random link blockage, the outage performance and effective throughput of the proposed transmit precoder design significantly outperforms several baseline scenarios, and results in more stable connectivity for highly reliable communication.
\end{abstract}

\section{INTRODUCTION}

To accommodate the surge of ever-increasing data-intensive wireless applications, millimeter-wave (mmWave) communication provides relatively large system bandwidth and the possibility of packing significantly large number of antenna elements for highly directional communication [1]. This is important to ensure link availability as well as to control interference to/from other users in dense deployments [2]. However, mmWave frequencies are more vulnerable to blockages due to higher penetration losses and reduced diffraction [3], which gives rise to unreliable communication and unstable connectivity. Further, frequent interruptions and longer duration of link blockage significantly degrade the quality of userexperience. To overcome such challenges, use of Coordinated Multi-Point (CoMP) transmission is imperative, where each user is concurrently connected to multiple base-station (BS) and receives information from the geographically separated transmitters for better communication robustness [4]-[9].

CoMP has been widely studied over past few years under the context of 4G systems [4]-[6]. Multi-point connectivity is also proposed in recent $3 \mathrm{GPP}$ specifications as one of the key requirements for upcoming 5G system [9]. Recent studies show the deployment of CoMP in the mmWave frequencies [7], [8]. In [7], the authors showed coverage is significantly improved by simultaneously serving a user with spatially distributed transmitters. The network coverage gain for the heterogeneous mmWave system was also confirmed in [8] using stochastic geometry tools. Despite the capacity and coverage improvements obtained by CoMP connectivity, as shown in [4]-[8], it is yet unclear how the concurrent transmission from spatially distributed transmitters should be designed to provide highly reliable mmWave communication.

It is well known that a system can provide any level of reliability by retransmitting the same message at various protocol levels, until receiver acknowledges correct reception, using a dedicated feedback channel [10]. However, in the presence of random link blockage and high path-loss at the mmWave frequencies, feedback links are equally unreliable and thus results in redundant retransmission of same messages. On the other hand, allowable latency determines strict upper limit on the number of retransmission attempts [11].

The loss of connection in the mmWave communication is mainly because of sudden blockage of the dominant link due to abrupt mobility, self-blockage or external blockers [3]. Accurate estimation of each blocker requires precise environment mapping and frequent Channel State Information (CSI) acquisition, which might results in significantly higher cooperation overhead and very difficult synchronization challenges. Thus, the current CoMP techniques [4]-[8] are not suitable for the stringent latency and reliability requirements of industrialgrade wireless communication and critical data applications.

Motivated by above concern, there is need to develop a robust and resilient transmission algorithm that can retain stable connectivity under the uncertainties of mmWave channels. To this end, we provide a novel Weighted Sum-Rate Maximization (WSRM) and Minimum User-Rate Maximization (MURM) design with the strong emphasis on system-level reliability, exploiting the multi-antenna spatial diversity with CoMP connectivity to optimize the downlink transmission rate. For the downlink precoder design, a conservative estimate of the available rate or SINR per user is formulated over all subset combinations of potentially blocked links. The original non-convex and challenging problems are approximated as tractable convex optimization problems leveraging the Successive Convex Approximation (SCA) framework and that is efficiently solved using standard of-the-shelf solvers. We further investigate and quantify the advantage of this solution in terms of achievable sum-rate and reliable connectivity.

\section{System MODEL}

We consider downlink in a multi-user multiple-input-singleoutput (MU-MISO) communication system with $B$ transmitters (BS) and $K$ single antenna receivers (users). The set of serving BSs is represented by $\mathcal{B}=\{1,2, \ldots, B\}$, set of active user indices is denoted as $\mathcal{K}=\{1,2, \ldots, K\}$ and serving set of BSs for $k^{t h}$ user is represented with $\mathcal{B}_{k}$, where $\mathcal{B}_{k} \subseteq \mathcal{B}$. Each BS is equipped with a uniform linear array (ULA) 


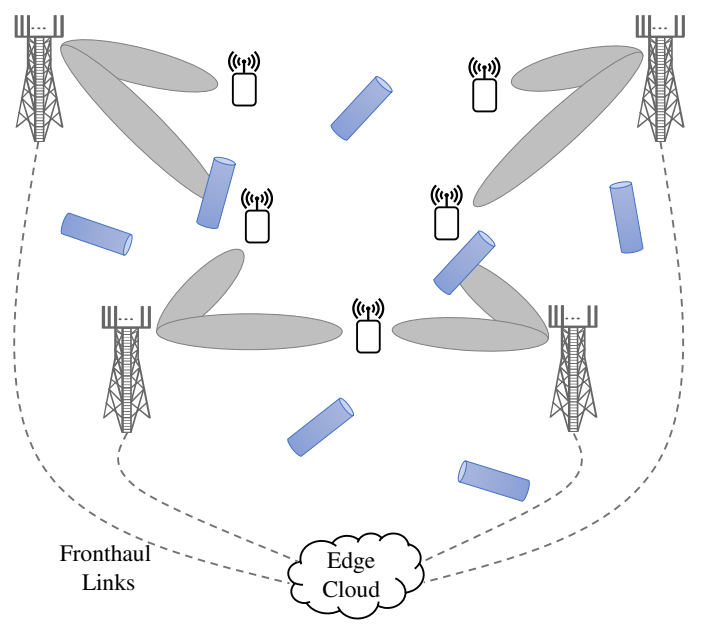

Fig. 1. Downlink communication system model showing transmitters (BSs) and receivers (users) with the LoS link and random blockers.

of $N_{t}$ isotropic antennas. Each antenna element is assumed to have $0 \mathrm{dBi}$ gain and $s=\lambda / 2$ spacing between any two adjacent elements, where $\lambda$ is the wavelength corresponding to the operating frequency.

While the problem formulation and proposed method are general and applicable to any architecture, in this paper, we restrict ourself to the case when each antenna is equipped with a dedicated baseband circuit to enable fully digital signal processing. For the future work, our aim is to include two-stage hybrid analog-digital architecture with the analog beamforming using long-term channel statistics followed by a less-complex digital precoding.

We consider CoMP connectivity, whereby, each user receives a coherently synchronous signal from all serving BSs. Further, all the transmissions are done using the same time and frequency channel. All serving BSs are connected to a edge cloud by the fronthaul links with finite capacity and zero delay, as shown in Fig. 1. The digital signal processing functionalities of each BS are transferred over a low latency connection to a common baseband processing unit (BBU), in the edge cloud. Furthermore, perfect estimation of available (non-blocked) CSI is assumed at the BBU for the downlink precoder design and resource allocation, whereby, each BS receives related information for the serving users, such as control and data signals, using the finite capacity fronthaul links.

\section{A. Channel Model}

Due to relatively low-scattering, higher path loss and spatially sparse nature of the mmWave channels compared to sub-6GHz frequency band, mmWave channel effectively have 'quasi'-optical propagation properties [1], [3], where the lineof-sight (LoS) link is the dominant path and mainly contributes to the communication. Thus, unblocked LoS path is highly desirable to initiate and maintain stable mmWave communications [3]. For simplicity, we neglect potential NLoS components and assume single-path MISO channel $\mathbf{h}_{b, k}$ between a BS-user $(b, k)$ pair, given by

$$
\mathbf{h}_{b, k}=g_{b, k} \mathbf{a}_{T}^{\mathrm{H}}\left(\phi_{b, k}\right), \forall b \in \mathcal{B}, \forall k \in \mathcal{K},
$$

where $g_{b, k}=\sqrt{N_{t}} v_{b, k} d_{b, k}^{-\varrho / 2}$, in which $v_{b, k}$ is the complex gain of the dominant path with zero-mean and unit-variance, $d_{b, k}$ denotes the distance between BS-user $(b, k)$ pair with the path-loss factor $\varrho$. The transmit array response vector of ULA is $\mathbf{a}_{T}\left(\phi_{b, k}\right) \in \mathbb{C}^{N_{t} \times 1}$ for the angle-of-departure (AoD) $\phi_{b, k}$.

We further assume that the presence of random blockers is not known at the time of precoder design. More specifically, the channel between any BS-user pair can have one of two states: LoS or blockage. Blockage state occurs when the dominant LoS path is blocked by any obstacle or the corresponding path-loss becomes sufficiently high to establish a communication link. Furthermore, even a mobile human blocker can block the dominant LoS link for approximately $500 \mathrm{msec}$ [3] and that can be equivalently modeled as $\mathbf{h}=0^{N_{t} \times 1}$. Finally, the channel between any BS-user pair $(b, k)$ can be model as

$$
\mathbf{h}_{b, k}= \begin{cases}\sqrt{N_{t}} v_{b, k} d_{b, k}^{-\varrho / 2} \mathbf{a}_{T}\left(\phi_{b, k}\right) & \text { if LoS state, } \\ 0^{N_{t} \times 1} & \text { if blockage state. }\end{cases}
$$

Since blockers are completely random, their position and orientation may change multiple times within the channel coherence interval. Therefore, it is difficult to estimate and/or predict the blockage state before each transmit interval. We assume that the transmit precoders at the BBU are designed assuming the LoS state. Thus, a system is in outage, if dominant LoS link is not available during the transmission, due to the random link blockage. It should be noted, a channel can also be in the blockage state during the estimation phase and available during data transmission. However, these links will not be included for the transmission. Thus, from the reliability prospective, we have to consider the case when channel is available during the estimation phase but not during the transmission, which is not a priori known at the BBU.

\section{B. Signal Model}

In this sub-section, we develop a generic signal model for the system shown in Fig. 1. The received signal at $k^{t h}$ user, $y_{k}$ is given by

$$
y_{k}=\sum_{b \in \mathcal{B}_{k}} \mathbf{h}_{b, k}^{\mathrm{H}} \mathbf{f}_{b, k} x_{k}+\sum_{k^{\prime} \in \mathcal{K} \backslash k} \sum_{b \in \mathcal{B}_{k^{\prime}}} \mathbf{h}_{b, k}^{\mathrm{H}} \mathbf{f}_{b, k^{\prime}} x_{k^{\prime}}+w_{k},
$$

where $\mathbf{h}_{b, k} \in \mathbb{C}^{N_{t} \times 1}$ is the channel between a BS-user $(b, k)$ pair. The data symbol $x_{k}$ has $\mathbb{E}\left[\left|x_{k}\right|^{2}\right]=1, w_{k} \in \mathcal{C N}\left(0, N_{o}\right)$ is circularly symmetric additive white Gaussian noise (AWGN) with power spectral density (PSD) of $N_{o}$. Finally, $\mathbf{f}_{b, k} \in \mathbb{C}^{N_{t} \times 1}$ represents the portion of the joint transmit precoder for $k^{\text {th }}$ user from $b^{t h}$ BS, designed by the BBU assuming full-CSI. The received SINR for the $k^{t h}$ user can be formulated as

$$
\gamma_{k}\left(\mathcal{B}_{k}\right)=\frac{\left|\sum_{b \in \mathcal{B}_{k}} \mathbf{h}_{b, k}^{\mathrm{H}} \mathbf{f}_{b, k}\right|^{2}}{\sum_{k^{\prime} \in \mathcal{K} \backslash k}\left|\sum_{b \in \mathcal{B}_{k^{\prime}}} \mathbf{h}_{b, k}^{\mathrm{H}} \mathbf{f}_{b, k^{\prime}}\right|^{2}+N_{0}} .
$$

\section{Problem Formulation}

The major goal of this work is to improve the system reliability for the robust transmission in the downlink with the objectives of WSRM and MURM. To this end, we need to obtain the optimal joint transmit precoder $\mathbf{F}=\left[\mathbf{f}_{1,1}, \mathbf{f}_{1,2}, \ldots, \mathbf{f}_{B, K}\right]$. 
The precoder design can be cast as the following WSRM problem as,

$$
\begin{aligned}
& \underset{\mathbf{F}, \tilde{\gamma}_{k}}{\operatorname{maximize}} \sum_{k \in \mathcal{K}} \delta_{k} \log \left(1+\tilde{\gamma}_{k}\right) \\
& \text { subject to } \gamma_{k}\left(\mathcal{B}_{k}^{\mathrm{c}}\right) \geq \tilde{\gamma}_{k} \quad \forall k, \mathcal{B}_{k}^{\mathrm{c}} \subseteq \mathcal{B}_{k},\left|\mathcal{B}_{k}^{\mathrm{c}}\right| \geq L \text {, } \\
& \sum_{k \in \mathcal{K}}\left\|\mathbf{f}_{b, k}\right\|^{2} \leq P_{b} \quad \forall b, \\
& \text { or MURM problem as, } \\
& \begin{array}{cl}
\underset{\mathbf{F}, \tilde{\gamma}_{k}}{\operatorname{maximize}} & \min _{k} \tilde{\gamma}_{k} \\
\text { subject to } & \gamma_{k}\left(\mathcal{B}_{k}^{\mathrm{c}}\right) \geq \tilde{\gamma}_{k} \quad \forall k, \quad \mathcal{B}_{k}^{\mathrm{c}} \subseteq \mathcal{B}_{k},\left|\mathcal{B}_{k}^{\mathrm{c}}\right| \geq L, \\
& \sum_{k \in \mathcal{K}}\left\|\mathbf{f}_{b, k}\right\|^{2} \leq P_{b} \quad \forall b,
\end{array}
\end{aligned}
$$

where $\delta_{k}$ is the priority weight corresponding to the rate of $k^{t h}$ user and $\gamma_{k}\left(\mathcal{B}_{k}^{c}\right)$ is defined as in (4). The constraints (5c) and $(6 \mathrm{c})$ restrict the total transmit power for each BS $b$ to be bounded by $P_{b}$. Constraints (5b) and (6b) are the lower bounds of the SINR as seen by each user. The SINR is computed over all possible subset combinations of size $\left|\mathcal{B}_{k}^{\mathrm{c}}\right| \geq L$ of the serving BSs. Thus, constraints (5b) and (6b) guarantee reliable connectivity, even if, at maximum, $L-\left|\mathcal{B}_{k}\right|$ coordinating BSs for user $k$ are unavailable due to random blockage, which is not known to the BBU during the downlink precoder design.

Problems (5) and (6) are non-convex, since $\gamma_{k}\left(\mathcal{B}_{k}^{\mathrm{c}}\right)$ cannot be handled directly in (5a) and (6a) as a convex constraint and no direct solution exists. In Section IV, we present an iterative algorithm by exploiting the convex approximation techniques.

\section{Reliability Via Multi-POINT CONNECTIVITY}

For improving the system reliability in CoMP-Joint Transmission (JT), the downlink precoder for each user $k$ is designed considering a smaller number of coordinating BSs $L$, than the actual number of BSs $\left|\mathcal{B}_{k}\right|$ serving the user. Since blockage event and position of blockers can not be known a priori, the BBU models the SINR over all possible subset combinations and uses the worst case estimate $\tilde{\gamma}_{k}$ in the objective for the downlink precoder design. For example, referring to Fig. 1, let $L=3$ and $\left|\mathcal{B}_{k}\right|=4$, then the subset $\overline{\mathcal{B}}_{k}$ includes the following BS indices

$$
\overline{\mathcal{B}}_{k}=\{\{1,2,3\},\{1,2,4\},\{1,3,4\},\{2,3,4\},\{1,2,3,4\}\},
$$

and $\tilde{\gamma}_{k}=\min \left(\gamma_{k}\left(\mathcal{B}_{k}^{\mathrm{c}}\right)\right)$. However, in actual, each user $k$ is always communicating with all $\mathcal{B}_{k}\left(\supseteq \mathcal{B}_{k}^{c}\right)$ BSs, unless the dominant LoS link between a BS-user pair is not available because of the blockage. Therefore, a user can be in outage only if less than $L$ links are available during the transmission phase. In contrary, if more than $L$ links were available, the actual achievable rate at the receiver would be somewhat larger than the assigned rate to each user.

Using the binomial distribution, we can model the success probability $\tilde{q}_{k}$ for $k^{t h}$ user as

$$
\tilde{q}_{k}\left(t ;\left|\mathcal{B}_{k}\right|, q_{b, k}\right)=\sum_{t=0}^{\left|\mathcal{B}_{k}\right|-L}\left(\begin{array}{c}
\left|\mathcal{B}_{k}\right| \\
t
\end{array}\right)\left(1-q_{b, k}\right)^{\left|\mathcal{B}_{k}\right|-t} q_{b, k}^{t}
$$

where $q_{b, k} \in[0,1]$ is the probability of a link is in blockage state. Since all user are independent, the system is in outage if any of the $K$ users is in outage. Thus, total outage is defined as

$$
\widetilde{P}_{\text {out }}=1-\prod_{k=1}^{K} \tilde{q}_{k}\left(t ;\left|\mathcal{B}_{k}\right|, q_{b, k}\right)
$$

From (5) and (6), we can observe the impact of parameter $L$ on system reliability and achievable transmit rate. For example, a use of smaller subset size will improve system reliability. However, it leads to lower SINR and hence will results in lower transmit rate to each user. Conversely, larger subset size results in higher SINR and thus higher transmit rate, but more susceptible to outage and hence results in less stable connectivity. Clearly, there is a trade-off between achievable transmit rate and reliable connectivity. Our goal here is to understand and quantify this trade-off.

\section{BEAMFORMER DESIGN}

In this section, we elaborate on finding a feasible solution for (5) and (6). To begin with, the non-convex SINR constraints in (5b) and (6b) can be handled by SCA technique, as shown in [12], where the authors provided SINR constraint approximation method for Coordinated Beamforming (CB) with global-CSI and no blockage. We extend this approach to take into consideration coherent multi-point transmission and provide novel grouping of multitude of potential interference conditions due to the blockage. The individual SINR constraint for each $\mathcal{B}_{k}^{c} \subseteq \mathcal{B}_{k}$ is solved as in [12]. The exact details are omitted due to lack of space. In the following, we reproduce main steps for brevity. The SINR expressions in (5b) and (6b) can be relaxed as

$$
\begin{aligned}
\gamma_{k}\left(\mathcal{B}_{k}^{\mathrm{c}}\right) & \leq \frac{\left|\sum_{b \in \mathcal{B}_{k}^{\mathrm{c}}} \mathbf{h}_{b, k}^{\mathrm{H}} \mathbf{f}_{b, k}\right|^{2}}{I_{k}(\mathcal{B})} \\
I_{k}(\mathcal{B}) & \geq \sum_{k^{\prime} \in \mathcal{K} \backslash k}\left|\sum_{b \in \mathcal{B}_{k}^{\mathrm{c}}} \mathbf{h}_{b, k}^{\mathrm{H}} \mathbf{f}_{b, k^{\prime}}\right|^{2}+N_{o},
\end{aligned}
$$

where (9) is the lower bound for $\gamma_{k}\left(\mathcal{B}_{k}^{\mathrm{c}}\right)$ seen by $k^{\text {th }}$ user and (10) is the upper bound of total interference plus noise. Without loss of generality, for the upper bound, we consider worst-case interference scenario. This will also simplify (5) and (6), as all the possible combination in $\overline{\mathcal{B}}_{k}$ will have same interference constraint. Even with this reformulation of (4), (5) and (6) are still non-convex because of (9) and cannot be handled directly. Therefore, we adopt a widely used approximation technique based on the SCA framework [12]. In SCA method, all non-convex constraints are replaced with a sequence of convex subsets that are iteratively solved until convergence. For the conciseness of mathematical representation, (9) can be rewritten as

$$
\gamma_{k}\left(\mathcal{B}_{k}^{\mathrm{c}}\right) \leq \frac{\left|\overline{\mathbf{h}}_{k}^{\mathrm{cH}} \overline{\mathbf{f}}_{k}\right|^{2}}{I_{k}(\mathcal{B})}
$$

where $\overline{\mathbf{f}}_{k}=\left[\mathbf{f}_{\mathcal{B}_{k}(1), k}^{\mathrm{T}}, \ldots, \mathbf{f}_{\mathcal{B}_{k}\left(\left|\mathcal{B}_{k}\right|\right), k}^{\mathrm{T}}\right]^{\mathrm{T}}$ is the stacked transmit beamformer. Finally, we denote the stacked channel corresponding to each subset combination for user $k$ as $\overline{\mathbf{h}}_{k}^{\mathrm{c}} \forall c$. For example, again referring to the Fig. 1, let $L=2,\left|\mathcal{B}_{k}\right|=4$ 
and $\mathcal{B}_{k}^{c}=\{1,3\}$ then the corresponding stacked channel $\overline{\mathbf{h}}_{k}^{c} \triangleq\left[\mathbf{h}_{1, k}, \mathbf{0}, \mathbf{h}_{3, k}, \mathbf{0}\right]$. The RHS of (11) is convex (quadraticover-linear) function, and, thus, can be bounded by first order Taylor approximation as

$$
\begin{gathered}
\mathcal{F}_{k}^{\mathrm{c}(i)}\left(\overline{\mathbf{f}}_{k}, I_{k}(\mathcal{B}) ; \overline{\mathbf{f}}_{k}^{(i)}, I_{k}^{(i)}(\mathcal{B})\right) \triangleq 2 \frac{\overline{\mathbf{f}}_{k}^{(i) \mathrm{H}} \overline{\mathbf{h}}_{k}^{\mathrm{c}} \overline{\mathbf{h}}_{k}^{\mathrm{cH}}\left(\overline{\mathbf{f}}_{k}-\overline{\mathbf{f}}_{k}^{(i)}\right)}{I_{k}^{(i)}(\mathcal{B})} \\
+\frac{\left|\overline{\mathbf{h}}_{k}^{\mathrm{cH}} \overline{\mathbf{f}}_{k}^{(i)}\right|^{2}}{I_{k}^{(i)}(\mathcal{B})}\left(1-\frac{I_{k}(\mathcal{B})-I_{k}^{(i)}(\mathcal{B})}{I_{k}^{(i)}(\mathcal{B})}\right) \leq \frac{\left|\overline{\mathbf{h}}_{k}^{\mathrm{cH}} \overline{\mathbf{f}}_{k}\right|^{2}}{I_{k}(\mathcal{B})}
\end{gathered}
$$

where the $\mathcal{F}_{k}^{\mathrm{c}(i)}\left(\overline{\mathbf{f}}_{k}, I_{k}(\mathcal{B})\right)$ is a under-estimator for the RHS of (11) with equality only at the operating point $\left\{\overline{\mathbf{f}}_{k}^{(i)}, I_{k}^{(i)}(\mathcal{B})\right\}$. The fixed operating points for the current iteration are updated from the solution of current SCA iteration. This is repeated until it convergence of the objective. After replacing constraints (5b) and (6b) with LHS of (12), the sub-problem for each SCA iteration can be efficiently solved, using existing convex optimization toolboxes, such as CVX [13].

\section{Simulation Results}

This section provide numerical results to validate the performance of proposed method. We analyze the impact of subset size $L$ on the outage performance and achievable throughput, as well as evaluate the trade-off between performance metrics.

\section{A. Simulation setup}

We consider downlink MU-MISO communication system with $K=4$ and $B=4$, where each BS is equipped with $N_{t}=\{4,16\}$ transmit antennas. BSs are placed at the corners of a square cell with the inter-site distance of 100 meters. We assume that users are randomly distributed within the coverage region, each user with different path gains and AoD with respect to each BS. An illustration of BS placement and randomly dropped users is shown in Fig. 1.

We consider full-cooperation JT scenario $\mathcal{B}_{k}=\mathcal{B} \forall k$, i.e., all BSs coherently serve all active users in the system. In practice, fronthaul link capacity and signaling overhead will limit the maximum number of active users to each BS. For simplicity, all BSs are assumed to have same maximum transmit power i.e., $P_{b}=P_{t} \forall b$, noise variance of $N_{o}=1$ and the carrier-frequency is set to $f_{c}=28 \mathrm{GHz}$. The signal-to-noiseratio (SNR) of $20 \mathrm{~dB}$ for each user is defined on the cell-edge from the closest BS. More precisely, SNR $=P_{t} / d_{e}^{-\varrho / 2} \forall b$, where $d_{e}^{-\varrho / 2}$ denotes the cell-edge distance with the path-loss factor $\varrho=2$. The problem is modeled using CVX [13] and all the results are averaged over 500 channel realizations.

\section{B. Probability of Outage}

The first results, illustrated in Fig. 2a and Fig. 2b, shows the outage performance as a function of increasing link blockage probability $q_{b, k}$, for WSRM and MURM, respectively. Outage event occurs if the downlink rate $R_{k}$ assigned at the transmitters is higher than the achievable rate $C_{k}$, for any user $k=1, \ldots, K$, given all available links, i.e., $P_{\text {out }} \triangleq \mathbf{P}\left\{R_{k}>C_{k}\right\} \forall k \in \mathcal{K}$.

It can be seen from Figs. $2 \mathrm{a}$ and $2 \mathrm{~b}$ that outage performance improves by decreasing the subset size $L$. Clearly, lower $L$

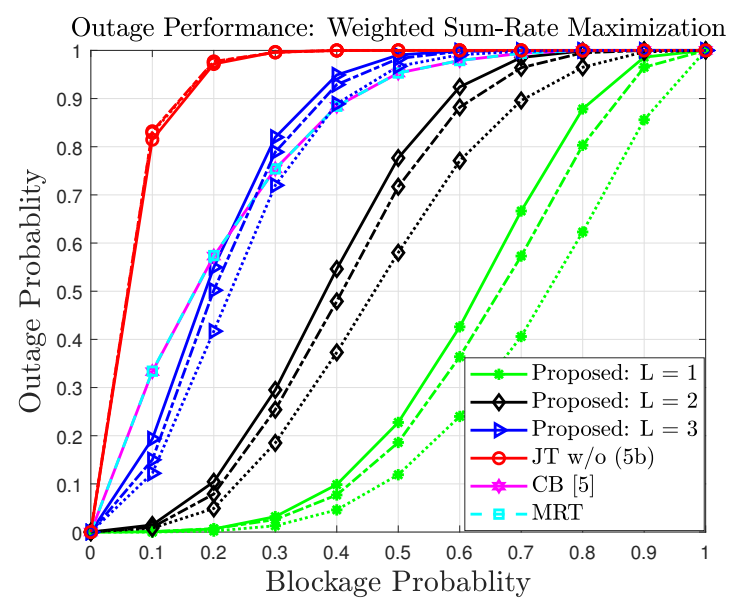

(a) WSRM

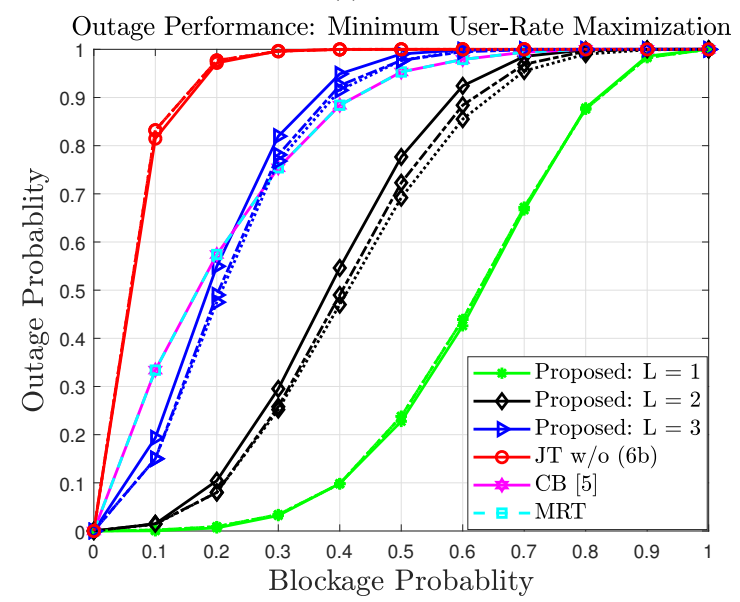

(b) MURM

Fig. 2. Outage performance for the (a) WSRM and (b) MURM problems as a function of increasing link blockage probability with theoretical formula (8) (solid) and simulations $N_{t}=4$ (dotted), $N_{t}=16$ (dash-dotted).

provides more stable connectivity and highly reliable communication. For example, the specific allocation with $L=1$ can withstand blockage up to a single active link. For a scenario with $N_{t}=4$, the outage performance with the WSRM appears slightly better, as the optimization problem solved at the BBU may end up assigning non-zero powers to only a subset of users, while all remaining users are assigned zero rate. In such a scenario, missing a link results in somewhat lower blockage than what is predicted by the theoretical formula (8). Furthermore, an increase in the transmit antennas at the BS also increases the beamforming gain. Thus, it is likely that all active users are assigned with some non-zero downlink rate, and, therefore, the simulated results with $N_{t}=16$ closely match to theoretical results obtained using (8). Contrarily, with the MURM approach, downlink precoder is designed such that all active users are always assigned equal transmit rate. This is because the downlink rate is always limited by the worst active user. Therefore, the simulated outage performance matches well with the theoretical curves for both $N_{t}=\{4,16\}$ as shown in Fig. 2b. However, our proposed method significantly outperforms the conventional JT, CB and Maximum Ratio Transmission (MRT) based beamforming. 


\section{Effective System Throughput}

Fig. 3a and Fig. 3b show the effective system throughput as a function of increasing blockage probability with identical weights $\delta_{k}=1 \forall k$ and $N_{t}=16$ for WSRM and MURM, respectively. Effective throughput $T_{e}$ is defined as $T_{e} \triangleq\left(1-P_{\text {out }}\right) R$, where $R=\sum_{k \in \mathcal{K}} a_{k} \log \left(1+\tilde{\gamma}_{k}\right)$ i.e., when all the active users successfully receive downlink data.

It can be seen that the system throughput decreases with reduced subset size $L$, when all dominant LoS links are available, i.e., link blockage probability $q_{b, k}=0, \forall(b, k)$. This behavior is expected as with smaller $L$, the aggregate SINR (rate) assigned for each user will be consequently lower. However, with increasing $q_{b, k}$, the effective throughput with $L=3$ quickly approaches zero due to the impact of outage. Clearly, there is trade-off between achievable system throughput and outage performance. More specifically, for a given $q_{b, k}$ and outage threshold, we can guarantee minimum achievable system throughput and vice-versa.

Furthermore, the achievable system throughput with the MURM approach is somewhat lower, as the BBU enforces equal rates for each transmit interval. In WSRM, on the other hand, users with different channel conditions are assigned varying user-specific rates leading to higher sum throughput. However, our proposed method provides more robust and stable connectivity, whereas with the convectional JT, CB and MRT methods, the sum-rate is exponentially decreasing with the small increase in link blockage probability.

\section{CONCLUSION}

In this paper, we studied reliable connectivity in mmWave wireless access by exploiting multi-antenna spatial diversity and coordinated multi-point transmission. We proposed a novel method for the optimization of the transmission rate, while considering link sensitivity in the mmWave frequencies. Our proposed approach is scalable to any arbitrary mmWave based multi-point configuration and dense deployment. Two different optimization criteria WSRM and MURM are considered and a novel SCA based algorithm is proposed to handle the nonconvex constraints. Simulation results manifested the robustness of proposed transmit precoder design in presence of random link blockage. It can be concluded that the outage performance and achievable sum-rate with the proposed method significantly outperform the baseline scenarios and results in a more stable connectivity for highly reliable communication.

\section{ACKNOWLEDGMENTS}

The research leading to these results has received funding from the Academy of Finland projects: Positioning-aided Reliably-connected Industrial Systems with Mobile mmWave Access (PRISMA) and 6Genesis Flagship (Grant No. 318927).

\section{REFERENCES}

[1] T. S. Rappaport et al., "Millimeter wave mobile communications for 5G cellular: It will work!" IEEE Access, vol. 1, pp. 335-349, 2013.

[2] J. G. Andrews et al., "What Will 5G Be?" IEEE J. Sel. Areas Commun., vol. 32, no. 6, pp. 1065-1082, Jun. 2014.

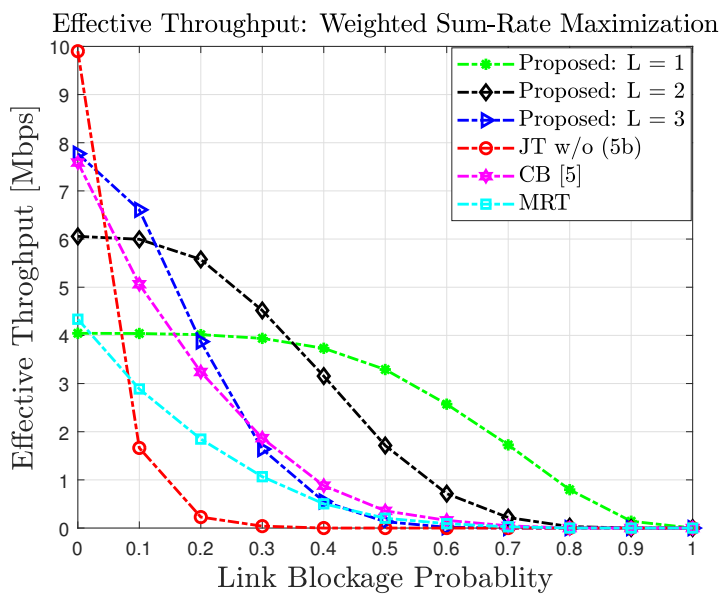

(a) WSRM

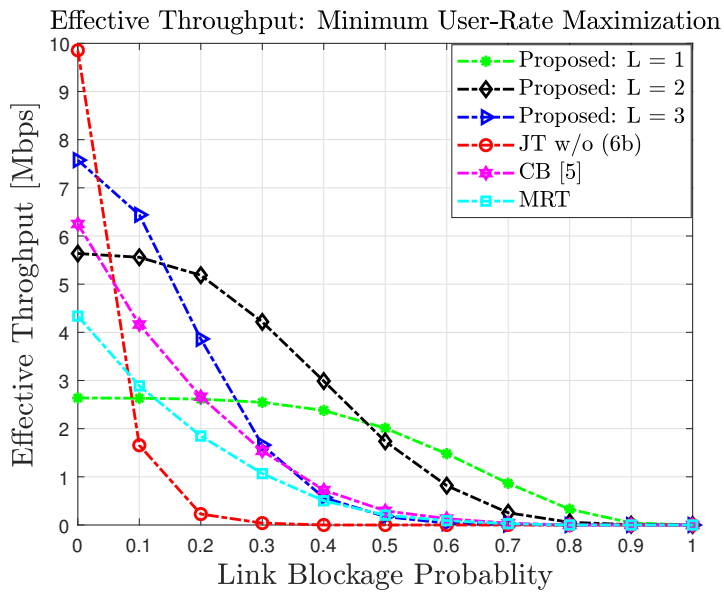

(b) MURM

Fig. 3. Effective throughput for the (a) WSRM and (b) MURM problems as a function increasing link blockage probability with $N_{t}=16$.

[3] G. R. MacCartney et al., "Rapid fading due to human blockage in pedestrian crowds at $5 \mathrm{G}$ millimeter-wave frequencies," in GLOBECOM 2017 - 2017 IEEE Global Communications Conference, 2017.

[4] A. Tölli, M. Codreanu, and M. Juntti, "Linear cooperative multiuser MIMO transceiver design with per BS power constraints," in 2007 IEEE International Conference on Communications, 2007.

[5] A. Tölli, H. Pennanen, and P. Komulainen, "On the value of coherent and coordinated multi-cell transmission," in 2009 IEEE International Conference on Communications Workshops, June 2009, pp. 1-5.

[6] R. Irmer et al., "Coordinated multipoint: Concepts, performance, and field trial results," IEEE Communications Magazine, vol. 49, no. 2, 2011.

[7] G. R. MacCartney et al., "Base station diversity propagation measurements at $73 \mathrm{GHz}$ millimeter-wave for $5 \mathrm{G}$ coordinated multipoint (CoMP) analysis," in 2017 IEEE Globecom Workshops (GC Wkshps), 2017.

[8] D. Maamari, N. Devroye, and D. Tuninetti, "Coverage in mmWave cellular networks with base station co-operation," IEEE Transactions on Wireless Communications, vol. 15, no. 4, pp. 2981-2994, April 2016.

[9] 3GPP TSG RAN Meeting \#82, TS 37.340, "NR; Multi-connectivity; Overall description (Release 15)," Rel. 15, Jun 2018.

[10] N. A. Johansson et al., "Radio access for ultra-reliable and lowlatency 5G communications," in 2015 IEEE International Conference on Communication Workshop (ICCW), June 2015, pp. 1184-1189.

[11] H. Shariatmadari, Z. Li, M. A. Uusitalo, S. Iraji, and R. Jantti, "Link adaptation design for ultra-reliable communications," in 2016 IEEE International Conference on Communications (ICC), 2016.

[12] G. Venkatraman, A. Tölli, M. Juntti, and L. N. Tran, "Traffic aware resource allocation schemes for multi-cell MIMO-OFDM systems," IEEE Transactions on Signal Processing, vol. 64, no. 11, 2016.

[13] M. Grant and S. Boyd, "CVX: Matlab software for disciplined convex programming, version 2.1," http://cvxr.com/cvx, Mar. 2014. 